in parts with cavities, the upper lobe containing one extending throughout its whole length, and communicating by the above-named openings with the pleura. The right lung was adherent at the apex. The upper lobe was full of caseous pneumonia, and contained a small cavity at the top. Caseous masses were found in the middle lobe, along its upper border, extending seemingly from certain centres. The lower lobe was much congested, and contained a few miliary tubercles. The heart weighed seven ounces and was fatty, but the valves were healthy. The liver was enlarged, of nutmeggy consistence, and weighed three pounds. The spleen was amyloid. The kidneys and other organs were healthy. This was a fair instance of scrofulous pneumonia, and exemplified the striking features of this variety of consumption, which may be summed up as follows.

I. The intensity of the pyrexia and rapid emaciation.

2. The acuteness of the disorganising processes, excavation quickly succeering consolidation.

3. The inflammatory nature of the processes, and the rarity of miliary tubercle.

4. The absence of fibrosis.

5. The occurrence of pneumothorax, owing to two causes:-I. The size of the cavities formed; 2. The absence of pleural adhesions.

6. The tendency of the disease to localise itself in the lungs, and the freedom of other organs, such as the intestines and the serous membranes, from secondary products.

\section{ON DRAINAGE IN OVARIOTOMY.*}

By GEORGE GRANVILLE BANTOCK, M.D., F.R.C.S.Ed., Surgeon to the Samaritan Free Hospital for Women and Children.

With the exhaustive works of Spencer Wells and Peaslee in our hands, it might be thought that there remained nothing new to be said on the subject of ovariotomy. There is one point, however, to which attention may be profitably drawn, and on which you will look in vain for any information in these works. I refer to the subject of drainage of the peritoneal cavity as one of the steps of the operation.

It may be said that most of the early operations in which the ends of the ligature were left hanging from the wound were examples. This arose from the erroneous ideas then prevailing as to the behaviour of a portion of ligatured tissue. It was universally believed up to a late period, and it is even now a matter of extensive belief, that sloughing of the distal portion must ensue ; and it is only within the last few years that it has been shown, both by direct experiment and by post mortem examination of persons who have been subjected to ovariotomy, that, if the ligature be cut off short and guarded against the putrefactive process, such a result is almost unknown.

In 1872-3, Dr. Marion Sims published a paper in the Neze York Medical fournal, in which he showed, from an analysis of a large num. ber of post mortem examinations, that, in the great majority of cases, death was due to the absorption of septic matter from the peritoneal cavity. The conditions were found so similar, and the symptoms preceding death presented such uniformity, that no other conclusion was possible. He further showed that, as in Mr. Spencer Wells's hands, this usually fatal train of symptoms had been checked by the evacuation, through the vagina, of a quantity of highly irritating and often offensive fluid. Hence, he argued, that if we could only prevent this accumulation, we should obviate a considerable number of deaths. To this end, he ventured on the heroic proposition "to puncture the cul-de-sac of the vagina behind the cervix uteri, and to pass a tube of some sort into the peritoneal cavity to drain off any effusion that may take place in the said cavity", and "to do this in every instance, whether there are adhesions or not". In illustration, he quotes five cases in which this proposition was acted on, and of which three were successful. On perusing these cases, one cannot be favourably impressed with the results, which are far from justifying the universal adoption of this procedure.

At a recent meeting of the Royal Medical and Chirurgical Society of London, Mr. Spencer Wells gave an analysis of "three hundred additional cases of ovariotomy", in which he did little more than allude to the subject of drainage. Nowhere, as I have said, will you find any detailed information on this subject, and we are thrown back on a few isolated cases which are difficult to find.

It will be my duty, then, to speak, first, of the cases that would appear to demand the application of this principle, with illustrative ex-

* Read in the Section of Obstetric Medicine at the Annual Meeting of the British Medical Association in Manchester, August 1877 . amples; secondly, of the modes of effecting the object in view ; and thirdly, to endeavour to indicate the most effective and least troublesome method.

First, as to the cases which appear to demand this proceeding :-I may at once say that the idea of draining the peritoneal cavity after a simple uncomplicated case, i.e., without adhesions and with a good pedicle, is not to be entertained. Speaking generally, I would say that whenever, in the course of operation, it becomes evident or probable that oozing of blood or serum, or both, must go on after closure of the wound, and especially when there has been an escape of ovarian fluid into the peritoneal cavity, then drainage will be demanded. This general proposition will be best enforced by illustrative cases. It has probably occurred to those who are in the habit of seeing, or have seen, much of ovariotomy, to regret that means had not been used to avoid that which has been revealed by a post mortem examination. This, at least, is my experience; and I cannot but regret the omission in the first case which proved fatal in my hands. The operation was performed under very unfavourable conditions. I had tapped the patient a week before. I removed twenty-three pints. This was followed by inflammation of the cyst. On the morning of the operation, the patient's temperature was ror deg. She died in four days of septicæmia. There was about a pint of very acrid serum with broken down blood in the peritoneal cavity. The clamp was used. In some cases, the pedicle has served as a drain, as in a similar case which I saw under Mr. Wells's care. Had I used the drainage-tube, it is probable the result would have been different.

CASE 1.-J. S., aged 25, was admitted into the Samaritan Free Hospital in February 1876 , under the care of Mr. Spencer Wells. A week afterwards, she was tapped. Inflammatory symptoms followed, but she recovered within three weeks, when she went to a convalescent home. She returned again on July 6th. The patient had lost much flesh ; tumour very multilocular; adhesions evident ; uterus drawn up almost out of the pelvis; altogether an unpromising case. Mr. Wells kindly offered me one of his cases, and I selected this as the most urgently demanding operation. I had arranged to operate at 9.30 A.M. on July 21 st, 1876 . On my arrival, a little before that time, I found that the patient had been seized some hours before with severe abdominal pain. She was then too tender for examination, but the tumour had lost some of its prominence; there was more fluctuation (superficial). Pulse 130; temperature, 100.4 deg. It was believed that rupture had taken place; yet I decided to proceed with the operation as a forlorn hope.

Operation.-On opening the peritoneum, a considerable quantity of ovarian fluid and thin colloid matter escaped. The tumour was first tapped and then broken up with the hand. As the mass was drawn out, the adherent mesentery was ligatured as it came into view, as well as one of the fimbriæ of the left Fallopian tube. Abundant, but not very firm, pelvic adhesions were then broken down, liberating the mass, which sprang from the right side by a pedicle about an inch long and two inches broad. The pedicle was transfixed and secured by a double ligature, of which the ends were cut off short. A long tail of omentum was found adhering to the pedicle close to the uterus. Two ligatures were applied, and division effected between. A portion of omentum adhering to the abdominal wall close to the incision was not interfered with. It required a great deal of sponging to clear away the serum and colloid matter from the pelvic cavity and from amongst the intestines, and considerable oozing of bloody serum went on. Under these circumstances, I deemed it advisable to put a glass tube through the bottom of the wound, reaching to the bottom of the utero-rectal cul-de-sac before closing the wound with seven sutures in the usual way.

An hour after the operation, the temperature was 100.8 deg., and the pulse 146. Eight hours afterwards, the temperature was $102 \mathrm{deg}$., and the iced water cap was applied. Bloody serum escaped, and was drawn off from the tube for four days -in all, about a pint; in the course of the fourth day, a piece of India-rubber tubing was substituted for the glass; and on the next day, the discharge having ceased, this was removed and the opening forthwith closed up. The patient left her room on the eleventh day, and the hospital on the twenty.fourth day.

CASE II.-M. H., aged 48 , mother of four children, was admitted into St. Bartholomew's Hospital in August 1875, under the care of Dr. Greenhalgh. A few days afterwards, the tumour burst; the fluid was absorbed; and, at the end of three weeks, the patient left the hospital very much reduced in size. She re-entered the hospital in June 1876 , and on the 26th Mr. Thomas Smith removed twenty-one pints of fluid by tapping. The case was considered too unfavourable for operation; and on August I4th she entered the Samaritan Hospital, where I tapped her for Mr. Wells, removing twenty-two pints, and leaving a larger mass than before. The hospital was about to be closed for the 
annual cleaning, and the patient was sent home. At the reopening of the hospital on October Ist, the patient returned, urgently requiring relief, and Mr. Wells kindly allowed me to keep the case. On October 4th, she measured 42 inches in circumference at the umbilicus, $9^{1 / 2}$ inches from the ensiform cartilage to the umbilicus, $101 / 2$ inches from the umbilicus to the symphysis pubis, $111 / 2$ inches from the umbilicus to the right anterior superior spine of the ilium, and 12 inches to the left. I tapped her of nineteen pints and a half of fluid, to relieve the dyspnœea and pain of distension. There remained a larger mass than before firmly adherent on the left side (groin); the uterus was drawn up above the pubes, behind which the os could just be felt, and the roof of the vagina was much stretched and funnel-shaped. It was impossible to make out the outline of the uterus, which appeared to be merged in the tumour. After consultation with Mr. Wells and Drs. Savage and Greenhalgh, it was decided to give the patient the benefit of the doubt - a doubtful benefit. Apart from the vaginal condition, the difference in the external measurements indicated extensive adhesions.

On October IIth, in the presence of Drs. Greenhalgh, Junker, and several others, my colleague Mr. Thornton assisting me, and Dr. Champneys administering bichloride of methylene, I made an incision five inches long in the usual situation. The tumour was found universally adherent anteriorly, and some difficulty was experienced in separating the peritoneum and cyst-wall. After breaking down the adhesions and setting the tumour free, the uterus was seen to be embedded in the mass, and raised above the level of the pubes. Three ligatures were applied to as many pieces of adhering omentum, and the tip of the appendix cæci was secured in the same way. The broken up tumour was drawn out as much as possible, but it obstinately remained adherent deep down in Douglas's pouch, and I was in doubt as to the possibility of separating it. While holding the mass on the stretch with the right hand, I pressed the tips of the fingers of the left on what appeared to be the line of union. It began to give way, and I went on peeling off the tumour from the posterior aspect of the broad ligaments and uterus until I reached the fundus uteri. Here the uterine tissues proper were torn, and free bleeding ensued. Having passed beyond the fundus, I broke through the cyst-wall where it was thin and nonvascular, and, having applied a ligature to each cornu, I cut the mass away. There now remained a flap of cyst-wall on each side, about the size of the palm of the hand. A deep fissure in the fundus, from which there was rather free bleeding, was brought together by a continuous suture of fine silk, and several small bleeding points were secured by fine ligatures. The left flap was cut away so as to fit on the back of the uterus, to which it was fixed by sutures along the fundus, small bleeding points having been secured as they appeared in the cut edge. The right flap was likewise cut away as low as possible, care being taken to secure some small vessels as they appeared. The peritoneal cavity was now cleared out, and there being no active bleeding, though considerable oozing, I passed a glass tube into Douglas's pouch and closed the wound. The operation lasted an hour and a half ; and, from the loss of blood and the severe character of the operation generally, it was the universal opinion that the patient had only a few hours to live.

Five hours afterwards, the bandage and dressings were found saturated with dark bloody serum, and about an ounce of almost pure blood was drawn from the tube. Temperature $100.7 \mathrm{deg}$. ; pulse 104 ; respirations 30. Next morning, in addition to the contents of the sponge, which was saturated, I drew from the tube about two drachms of dark bloody serum. At the end of two days and a half, I obtained a small blood-clot. Temperature $103 \mathrm{deg}$. The iced water cap was put on. For the next hour, the temperature continued to rise, until it reached ro3. $6 \mathrm{deg}$. In fourteen hours, it stood at $99.8 \mathrm{deg}$., and the cap was removed. The temperature again rising, it was reapplied; and, with several changes, it was finally removed on the fifth day. The discharge from the tube continued, though in gradually diminishing quantity ; portions of clot were obtained on the fourth and fifth days ; on the sixth day, only about a drachm of pale red serum was obtained a each dressing, and the glass tube was withdrawn, its place being taken by a piece of India-rubber tubing of about half the diameter. This was removed the next day. On the eighth day, the opening was closed ; on the eleventh day, the patient was outside the bed; and on the seventeenth day, she went to a convalescent home. She is now in excellent health.

CASE III.-Mrs. C., aged 27, mother of four children, the youngest of whom was under two years, was admitted into the Samaritan Free Hospital on October 3 Ist, 1876, under my care, with a very large multilocular tumour. She measured $44 \mathrm{1} / 2$ inches in circumference at the umbilicus, $13 \frac{1}{2}$ inches from the ensiform cartilage to the umbilicus, 9 inches from the umbilicus to the pubes, and II inches from the umbilicus to the anterior superior spine of the ilium. (I mayhere again call attention to these differences of measurement as a sure indication of parietal adhesions.) The tumour was exposed by an incision five inches long, afterwards extended upwards to about eight inches. Adhesions were very firm over the whole anterior and lateral aspects of the tumour, from a little above the pubes to the ensiform cartilage. They were broken down with difficulty. On the right side, near the brim of the pelvis, there was a band of adhesion about an inch and a half broad, which I could not break through. It was ligatured. Four ligatures were applied to as many portions of adherent mesentery; and the pedicle, which was from two to three inches long, was treated in the same way. The tumour weighed fifty pounds, and the operation occupied about an hour. In consequence of the very extensive adhesions and the continuance of oozing, I deemed it advisable to use a drainagetube. About thirty ounces of serum, at first bloody, finally becoming colourless, were obtained; the tube was removed after four days and three quarters; the wound healed well; the opening closed within thirty-six hours after the removal of the tube, and the bowels acted on the eighth day. Her convalescence was retarded by the appearance of a large induration above and to the left of the umbilicus, threatening an abscess in the abdominal walls. It, however, gradually disappeared under treatment, and the patient left the hospital at the end of a month. She is now quite well.

CASE IV. - Miss C. U., aged 27, was admitted into the Samaritan Free Hospital on January Ist, 1877 , with a multilocular ovarian tumour of about two years' growth. On the night of January 5th-6th, the cyst ruptured. This was followed by symptoms of peritonitis. On the 8th, the temperature was 104 deg., and pulse 142 at 9 A.M. For a fortnight, the patient was so ill that death was feared; the abdomen became very tympanitic, tongue dry, red, and glazed, with troublesome sickness and obstinate constipation; and she lived on small quantities of milk, beef-tea, chicken-broth, gruel, and latterly a little bread and butter as the most solid food.

On the eighteenth day, her morning temperature was 98.6 deg., but the evening temperature rose to 102 deg.; and at 9 P.M., it stood at IOI deg.; pulse 120; the tongue had become a little moist; the bowels had been well moved by frequent small doses of colocynth and henbane; but the tympanites remained. A line drawn from the right hypochondrium to the inner end of the left Poupart's ligament, pretty nearly straight, divided the abdomen into two parts, of which the left was very tympanitic, and the right wholly dull on percussion. I determined to give her the chance afforded by an operation, as otherwise her death could not be far off. The patient readily assented to my proposition.

Nineteenth day, 9 A.M. Temperature, 99.4 ; pulse 112. Noon temperature, 100 deg. The operation was begun at 2.45 , in the presence of Drs. Gage Brown and Baxter Forman, who were interested in the case, and several other gentlemen. My colleague Mr. Thornton assisted me, and Dr. Champneys administered the anæsthetic. On dividing the abdominal wall, I found the peritoneum and cyst-wall intimately adherent, and I had to open the tumour at once, giving exit to a dark brown viscid fluid. Having then enlarged the opening and to a duated the greater portion of the fluid contents, I succeeded in separating the cyst-wall from the peritoneum for about an inch in breadth on each side. On these flaps, I fixed a pair of vulsellum-forceps, by which I was able to steady the tumour while I introduced my hand and broke up the mass. This done, I then proceeded to break down the parietal adhesions, which were universal, recent, and very vascular. A small piece of omentum was secured by ligature as soon as it came into view. I now passed my hand behind and around the tumour, rupturing adhesions to the intestines until it was set free. The pedicle, about two inches long and rounded, cord-like, about the thickness of a medium sized finger, was secured by a single ligature, and the mass was cut away. The transverse and descending colon, considerably distended, was now seen to be adherent to the abdominal wall, in a diagonal direction from right to left downwards, and close to the umbilicus and the top of the wound. From this down to the pelvis, an unbroken surface was presented, like the inside of a cocoanut-shell, formed by the adhesion of coils of small intestine to one another and to the colon, where they lay in contact with the left posterior aspect of the tumour; and the floor of the cavity was formed by the adhesion of the uterus and right broad ligament to the adjacent structures. Out of the floor of the cavity 'thus formed, I took a handful of firm colloid matter, having a very peculiar odour. High up on the right side, I could get amongst the intestines, which, although adhering to the tumour, were free from one another. To a less extent, I could get down into the left iliac fossa. Douglas's pouch was obliterated. There was free oozing from the surfaces of the intestines where the adhesions were ruptured, and one point near the bottom of the cavity required a fine ligature. A glass tube was finally inserted, and the 
wound closed in the usual way with seven sutures. The tumour weighed eleven pounds.

Two hours after the operation, the temperature was ror.4 deg., and pulse 120 . In six hours, the temperature had risen to 102.8 deg., pulse 144, respirations 24 ; and the iced water cap was put on. The bandage and dressings were saturated with red serum, of which I obtained about an ounce and a half from the tube and sponge. In twelve hours, the dressings had to be changed again, but the discharge was less free. Temperature 101.6 deg.

Suffice it to say that the discharge rapidly diminished, becoming paler ; flatus passed in about thirty hours; the tube was removed after sixty-five hours; two stitches were removed on the third day, and all were out on the sixth, the wound quite healed. The iced cap was kept on continuously for about fifty hours, when the temperature had fallen to 99.2 deg., pulse I03; it was resorted to for short periods for four days more, and was finally removed with the temperature at $98.6 \mathrm{deg}$. The bowels acted on the seventh day, after taking pills of a grain of compound extract of colocynth and one-sixth of a grain of extract of belladonna, and having an enema of soapy water. The patient was out of bed on the fourteenth day, and left the hospital on the twenty-sixth day, walking as sprightly as if nothing had happened.

CASE v. - M. A. W., aged I 5 , was sent from the camp at Aldershot by Staff-Surgeons A. F. Churchill and Alcock to the Samaritan Free Hospital on January 12th, I 877 . Her temperature at 5 P.M. was 101. 6 deg.; pulse 136. She looked much under age, and was greatly emaciated. There was great tenderness in the tumour, which felt very solid, and moved freely in a considerable amount of ascitic fluid. The circumference at the umbilicus was twenty-eight inches. The skin was harsh and dry ; the urine pale and free. On the third day, I gave her five minims of tincture of perchloride of iron three times daily; and, after some days, I increased the dose to ten minims. The urine became much more free, from three to four pints daily, very pale ; specific gravity 1008 ; no albumen. At the end of a fortnight, the ascitic fluid had disappeared; the tumour was but slightly tender; the maximum daily tem. perature exceeded 100 deg. ; and on the eighteenth day, at 9 P.M., it was 100.6 deg. ; pulse 140 . It was now evident that no more improvement could be expected, and that since the disappearance of the ascitic fluid, the tumour was becoming adherent to the parietes. I, therefore, decided to operate next day.

January 3 Ist, 9.30 A.M. Bichloride of methylene was administered by Dr. Percy Boulton, and Mr. Thornton assisted me, in the presence of several visitors. As I anticipated, the tumour was found universally adherent to the parietes and adjacent structures. The adhesions were evidently recent, as shown by their friability and excessive vascularity. The trocar thrust into the tumour drew no fluid, and I had to enlarge the incision to about five inches. The tumour was now turned out entire, bringing into view a thick pedicle about two inches broad and three inches long. The pedicle passed into the tumour half way up its left face, and appeared as a broad thick band, non-adherent, though in close apposition. A double ligature was applied, and the mass was cut away. A coil of small intestine adhered to the abdominal parietes just above the level of the left iliac crest. There was considerable sanguineous oozing from the ruptured adhesions in Douglas's pouch. The insertion of a drainage-tube and the closure of the wound by ten silk sutures completed the operation. The tumour was dermoid, and from it I obtained these specimens of bone. (The specimens of bone were shown.) There had been hæmorrhage into one or more of the larger cysts situated at the back of the tumour ; and this was, no doubt, the cause of the inflammatory action set up.

Half-an-hour before the operation, the temperature was $99.6 \mathrm{deg}$. (pulse 140); and an hour after, it was 99 deg. In six hours, I removed the dressings, which were saturated with red serum, and three drachms of dark bloody fluid were obtained from the tube. The tem. perature had then risen to 102 deg., and the iced water cap was put on. An hour later, the temperature was 102.4 deg., and the pulse was 172, and respirations only 16 . For eight hours, the temperature stood steady at 102.6 deg. ; but in two hours later it had fallen to ror. 8 deg., and it continued to fall until at midnight (thirty-two hours) it stood at 100 deg., and the pulse had fallen to 132 . The temperature again rose gradually in the next ten hours up to $102.3 \mathrm{deg}$., with a pulse of 148 ; and during eleven hours it fell as slowly to $100 \mathrm{deg}$. From this time, it gave no more anxiety, but the pulse would not fall below 130 , and often ran up to I44. The iced water cap was finally removed on the fifth day. In all, about half-a-pint of serum escaped; the tube was removed after sixty-seven hours, when the serum was of a pale brown colcur and inc dorcus. None escaped after this, and the opening closed in a few hours. Several small clots were removed from the tube in the process of cmptying it. On the third day, she passed six pints and a half of urine of srecific gravity 1008, of alkaline reaction, and containing one-fourth albumen. The subsequent history of the case does not bear on the question under consideration; and I have only to say that, in consequence of the formation of an abscess in the wound and the condition of the urine, the patient was not able to leave the hospital till the forty-first day. She is now in very fair health.

These five cases, presenting such varying and unpromising conditions, abundantly prove the great service rendered by the drainage-tube. I do not hesitate to say that, but for this, the result must have been different. I call particular attention to Case II, in which the whole posterior surface of the uterus was in a raw state, with not less than a dozen ligatures, large and small, in its immediate neighbourhood. It was the general impression in the room that this patient had only a few hours to live ; but we have seen that, by the early removal of the blood and serum, she was able to make an excellent recovery. But the most remarkable recovery was that of Case IV, in which the intestines were glued together by recent peritonitis.

It is now time to speak of the mode by which the drainage is effected. Dr. Marion Sims recommended that the drainage should be effected through the recto-uterine cul-de-sac and vagina; but the perusal of his cases will not impress one favourably. The difficulty of keeping the tube pervious and of preventing putrefaction is almost insuperable. If we could contrive a method by which we could convert the rectouterine pouch into the form of a funnel, no doubt this would prove the most efficient. But at present I am unable to see my way to this. Dr. Marion Sims's cases are not encouraging, for they show us the great difficulties attending it, even in his skilful hands. He figures an instrument, like a bivalve speculum, which be recommends, but which he had not then submitted to the test of experiment. Kœberle was, I believe, the first to employ a glass tube communicating through the lower end of the wound with Douglas's pouch. This tube was closed at the lower extremity, which in shape was rounded and pointed, and it was pierced throughout its length with a number of small holes. The latter I always regarded as a mistake, as it was not only inconvenient for removing the fluid collected in the tube, but admitted air too freely, and did not confine the fluid. It is to Dr. Keith that we are indebted for the form of tube which I now show you, and which appears to me to answer every purpose. It is made of various sizes as to length and diameter, and its peculiarities are that it is pierced for about an inch only of its lower end, that this is open, and that at the other end it has a shoulder to prevent its slipping into the peritoneal cavity. The fluid is thus drawn from the lowest part of the peritoneal cavity, and the air comes into contact only with the surface of the fluid in the tube.

To my mind, this tube is at once the most simple and efficient method yet attempted. It is managed in this way. After all actively bleeding points are secured and the sutures are all passed, Douglas's pouch is finally cleaned out, and, while with one hand the intestines are kept out of the way and the fingers serve to guide the tube, it is passed down to the bottom of the pouch between two of the sutures. Care must be taken that the tube maintains a perpendicular position. The sutures are now tied, a cap-shaped sponge wrung out of a solution of carbolic acid (one to twenty), is placed over the tube, and the dressing is completed. It is important to perform pressure uniformly over the abdomen, so that the fluid as fast as it is poured out is forced into the tube. Sickness soon after the operation is rather desirable than otherwise, as it prevents partial collections which are very apt to form from the union of raw surfaces which is inevitable in the case of torn adhesions. Immediately on the appearance of any discharge in the dressings, these should be changed. Under any circumstances, the first dressing should be done within from four to six hours. The sponge is well washed out and recharged with carbolic acid, and the contents of the tube are removed by means of a glass syringe, over the nozzle of which is drawn a piece of India-rubber tubing a little longer than the glass tube. The frequency of the dressing after this will depend on the amount of discharge ; but it will be well to repeat it at intervals of six or eight hours. At first, the serum contains more or less blood; but gradually becomes paler and paler, and diminishes in quantity. As soon as the discharge becomes of a pale colour and is so far reduced in quantity that not more than half a drachm or a drachm can be obtained from the tube and none has overflowed into the sponge, the tube may be removed. This usually happens about the fourth or fifth day. It is a good precaution to substitute a piece of India-rubber tubing for a few hours. If no fluid be obtained, or only a few drops, this also may be removed. The opening now closes in a few hours. Should, however, the general condition of the patient be in any degree unsatisfactory, it will be well to wait until the symptoms have subsided. The longest period to which the drainage was carried on in these cases was five days. This contrasts very favourably with Dr. Marion Sims's cases. In his first case, the drainage was continued for a fortnight; in the second, for six weeks ; in the third, the drainage was a failure, chiefly 
from the mode adopted ; in the fourth, for about a month ; and in the fifth, it again failed.

One great difficulty in drainage through the vagina will be that of preventing putrefaction. In not one of my cases was there even an approach to this.

It is thus an easy matter to insure drainage of the utero-rectal pouch; but the difficulty is to prevent partial accumulations. Since the above was written, I have had another case in which a collection of bloody serum took place between the liver and the parietes. The case was one of suppurating ovarian tumour intimately adhering to the parietes extending high up between the liver and the ribs. There was no sickness after the operation. On the third and fourth days, the temperature was normal, and the tube was removed on the third day. On the fifth day, the temperature began to rise; and she died within twenty hours, with all the symptoms of septicæmia. The post mortem examination showed that the drainage of Douglas's pouch was perfect, and there was no indication that the tube had been used. The liver was adherent to the raw surface in apposition, and behind this there was collected a small quantity of decomposed bloody serum, under two ounces, which had produced death. But for this small collection, the result would have been perfect, as shown by the examination.

How to prevent these partial accumulations is the only remaining difficulty ; but this paper has already attained such a length, that I must not further detain you.

\section{ON SOME POINTS IN THE ART OF PRESCRIBING FOR CHILDREN.*}

By ROBERT FARQUHARSON, M.D., F.R.C.P., Lecturer on Materia Medica at St. Mary's Hospital Medical School; etc.

I VENTURE to put before you a few practical observations on some points in the art of prescribing for children, because the subject is one which has hardly yet been treated on a sufficiently comprehensive basis. Much valuable but scattered information may be gleaned from the pages of contemporary literature, and much of what I am about to say has been said before; but it seems to me that some little service may be rendered by weaving these threads of knowledge into something of a more connected whole, and obtaining the opinion of some of those experienced physicians who have devoted themselves to the diseases of the very young.

Time, however, will not permit me to do more than touch, and that briefly, upon one point in connection with a subject which is really a large one, and to lay before you some facts and ideas on dosage; and here, again, I must once more subdivide, and take only a small section of a great therapeutical question, whose importance has only very recently begun to acquire that general appreciation which it eminently deserves. I might well be tempted to invite you to join with me in some reflections as to the comparative efficacy of the occasional large or the oft-repeated minute dose-a question which must before long become one of the most pressing in the materia medica; or it might be interesting to inquire as to the desirability or otherwise of inducing the physiological effects of drugs for the relief of pathological conditions; but at this time I mean to restrict myself simply to this proposition-the difference between children and adults in respect of the quantities of various drugs which may be taken, not only with actual impunity, but with absolute benefit.

Now, systematic works have too often not only ignored the teachings of Ringer, Fuller, and other modern investigators, but have done much to hamper and confuse our knowledge in this direction by laying down the law that children necessarily require much smaller doses of most of our more active drugs than adults; and we, therefore, see in books on materia medica, as well as on children's diseases, elaborate tables setting forth the quantities to be prescribed with safety at different periods of early life. Some years ago, and possibly even now, a student would run a good chance of being afforded the opportunity of continuing his studies, were he to tell his examiners that a child can take a dose of belladonna with impunity which would probably induce physiological symptoms in the adult; and, as a natural consequence of this mode of teaching, great timidity in practice has resulted ; and that this may be a positive evil requires but little refiection to show. If a dose of a particular remedy be too small to effect the purpose for which it is ordered, it is much more likely to do harm than good. Thus an insufficient purgative merely irritates the patient's bowels without giving relief; too small an opiate excites the nervous

* Read before the Medica Section at the Annual Meeting of the British Medical Association in Manchester, August 1877 . system and banishes that sleep which it was intended to attract; and numerous other instances will readily occur in illustration of a statement which hardly requires such confirmation.

Granted, then, the importance of administering our remedy in doses sufficient to produce their full remedial effect, I shall lay down, as my first and only proposition, that children require doses of many medicines quite as large as those which are commonly ordered for persons of mature age. Now, when I speak of children, I shall not refer to mere infants, whose tender organisation and sensitive organs and functions require special consideration from a therapeutical point of view. Thus the yielding nature of their skulls, admitting as it must of wide differences in the proportion of cerebral blood, no less than the natural tendency to sleep at that early age, plainly indicate caution in the use of narcotics. Purgatives and various other remedies must then be used with caution, or we may initiate an irritable condition of stomach and bowels which all our skill may not readily remove. In dealing with general principles, therefore, let it be understood that I refer to children over one year in age ; and, perhaps, before beginning the consideration of special instances in favour of my views, I may briefly touch upon the explanations which most naturally suggest themselves of the peculiarity which forms the excuse for my remarks. In prescribing for adults, we are frequently annoyed by the very various results obtained in different persons from a precisely identical quantity of a particular drug. Thus, one patient will develope a copious crop of acne from a few grains of bromide of potassium, whilst another can take ounces without such effect. Another will be salivated by a small quantity of mercury, or be unable to swallow quinine without uncomfortable nervous symptoms or a specially irritable rash. Children, however, do not present in anything like the same degree these special peculiarities of idiosyncrasy; the effects of medicines are pretty constant in their case, and we may generally anticipate the satisfaction of finding that our remedy has acted as we wished, and without any of that excess or eccentricity of action which too often brings undeserved discredit on the medical man. The reason which tells us why young children bear heavy doses of potent medicines must also cover this difference from their elders, and we might at once shut up further inquiry by concealing ourselves behind the dense cloak of ignorance implied in the assumed fact of an ultimate difference of constitution. But, true as this may be as an abstract proposition, we must look a little deeper, and ask, in the first place, whether some peculiarity of digestion may not come to our aid, and whether infants may not emulate some of the lower animals in the power which they possess of neutralising or destroying poisonous principles, as rabbits harmlessly browse on belladonna, and pigeons baffle the deadly action of strychnia, etc. But of such powers in the human being, at any period of life, we have no shadow of proof, presumptive or otherwise ; and it is probable that remedies reach the blood of children in the regular way, and through the same chain of physiological processes as in the case of adults. So we must again go forth in search of our explanation; and I think we may find some approach to it, at all events, in the view that, in consequence of the rapid growth taking place in the body during early life, the blood and tissues are in a condition of specially active destruction and renovation. Drugs, such as the metals, which probably combine with the albumen of the circulating fluid, are here rapidly cast out of the system. Other remedies, which act more particularly on the nervous system, are cast out with effete matters before they have had full time to produce their physiological effects, or, at all events, before these effects have attained to anything like completeness. Thus we do not often find developed in children that accumulation which occasionally, if rarely, is observed in patients of older growth, because the drug is removed before it can produce that continuous and ever-intensifying influence on the nervous system which eventually finds expression in what we may call a discharge.

So much, therefore, for my explanation, such as it is, of the facts which $\mathrm{J}$ shall now proceed briefly to lay before y.u.

Now, in the first place, I am bound, of course, to confirm the usual opinion of the dangers of opium in very early childhood; and it is not long since I saw an infant of eight minths nearly narcotised to death by six two-minim doses spread over two days. But those within the period of life which I have selected for consideration can bear mode. rate quantities, and chloral seems always well borne. For instance, I have lately had under treatment a little rickety girl suffering from recurring attacks of laryngismus stridulus, to whom three and a half grains were given with benefit thrice daily. The same patient took ten, and finally fifteen, grains of bromide of potassium, before any beneficial effect was attained; and I have always observed that this drug is well taken by children. Twenty and thirty grains have been no uncommon dose to reach in patients of from eight to ten suffering from epileptic seizures, and in them I have never observed any symptoms of bromism. 